\title{
La Tombe du Navire de Tarquinia et le navire étrusque de commerce
}

\author{
Jacques Bonnin \\ Ingénieur des Arts et Manufactures
}

\section{Introduction}

Peuple d'industriels et de commerçants, les Etrusques ont connu leur apogée, au milieu du millénaire qui précède notre ère, en particulier grâce à leurs échanges avec leurs voisins. Sur des territoires, à une époque, oủ le transport terrestre était tout à la fois moins efficace et plus dispendieux que le transport maritime, ils n'ont pas manqué de tirer parti de leur domination sur mer - qualifiée de thalassocratie - pour assurer leur commerce par voie maritime.

Or, nous sommes très mal renseignés sur leurs navires de commerce, encore moins souvent représentés que ceux des Grecs - leurs rivaux dans le sud de la péninsule italienne.

Par chance, la collaboration étroite et fructueuse apportée par les scientifiques à la recherche archéologique, dans le cadre de la Fondation Lerici, a permis, il y a une trentaine d'années, la découverte d'une tombe abritant une représentation unique d'un grand navire de commerce : la "Tomba della Nave "Tombe du Navire - sur le plateau des Monterozzi, dominant Tarquinia.

Très tôt, les fresques de cette tombe ont été déposées et transférées au musée archéologique de Tarquinia; les représentations encore visibles de cette tombe, ainsi que de 36 autres, découvertes lors de la même série de recherches, ont été publiées dès 1966, sous l'égide de la Fondation Lerici, dans un remarquable ouvrage dû à Mario Moretti [21].

Il nous a paru intéressant, à la lumière de ces documents ainsi que d'autres ayant trait au même sujet, de tenter de préciser quelques aspects relatifs aux navires de commerce étrusques à l'époque de la décoration de cette tombe, c'està-dire, comme nous le verrons, au milieu du $\mathrm{v}^{e}$ siècle avant notre ère. Auparavant, nous rappellerons les éléments essentiels de cette recherche archéologique scientifique, ainsi qu'une description de la décoration picturale de cette tombe, plus spécialement développée en ce qui concerne la représentation du navire.
2. La Tombe du Navire dans son contexte de fouilles et de publications

\section{I. "Nuovi Monumenti della Pittura Etrusca " : l'ouvra- ge, son auteur}

L'ouvrage [21] de Mario Moretti « Nuovi Monumenti della Pittura Etrusca " (Milano, 1966 - tiré à 3000 exemplaires) rassemble les décorations picturales de 37 tombes découvertes à partir de 1958 . L'abondance des découvertes à cette époque justifiait une telle publication, en ce qu'elle augmentait dans de fortes proportions l'iconographie étrusque, enrichissant d'autant nos moyens d'appréhension de la culture de ce peuple.

L'auteur, Mario Moretti, Surintendant aux Antiquités d'Etrurie Méridionale, était hautement qualifié pour cette présentation. Il a assorti chaque tombe d'un texte assez bref (dépassant rarement une page). En tête de [21] figurent un avant-propos de P. Gamacchio et une intéressante présentation du professeur Massimo Pallottino. Puis l'introduction de M. Moretti donne l'historique des fouilles, que suivent les caractéristiques et la signification de la peinture funéraire étrusque, avant de passer en revue celles qui sont présentées dans l'ouvrage.

\subsection{La Fondation Lerici et la prospection archéologique scientifique}

La découverte de très nombreuses tombes, et l'exploration de nombreuses tombes décorées à fresque, ne sont pas le fruit du hasard, mais d'une étude systématique entreprise par la Fondation Lerici. Carlo Maurizio Lerici, ingénieur, a voulu mettre

\section{The Tomb of the Ship in Tarquinia, and the Etruscan cargo ship}

The discovery, due to the use of highly elaborate prospection methods, of a decorated etruscan tomb showing, nearly 2 centuries ago, a large cargo ship, brings valuable information on tyrrhenian navy at the time of its apogee and just after.

This unique picture, compared to those of ships of neighbour commercial countries, to wreckages and to their freights, to ancient texts, allows us to improve our knowledge of etruscan maritime trade and of ships which ensured it, in a geographical context where, for a long time, "mare nostrum " was the most efficient link.

\section{LA HOUILLE BLANCHE/N 6-1991}




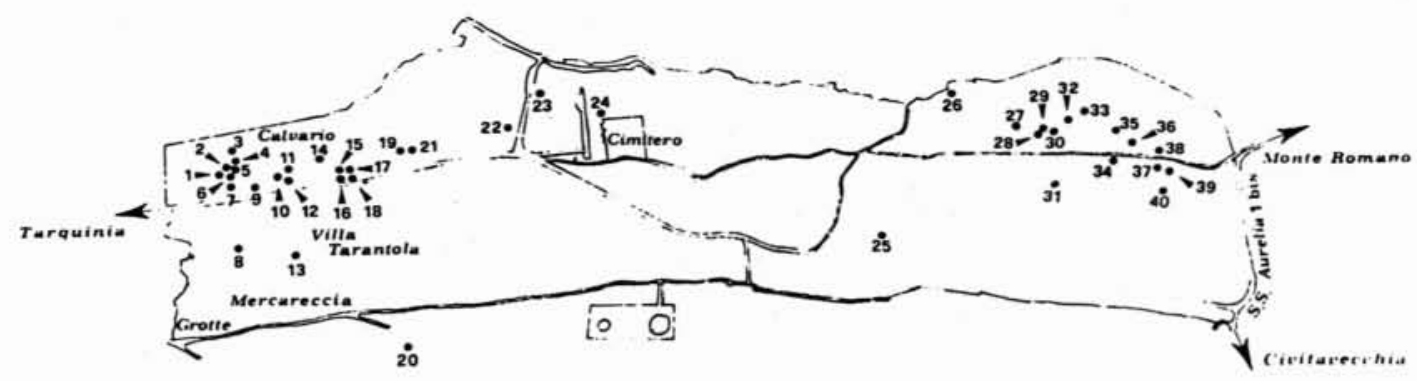

TARQUINIA: pianta della necropoli di Monteroza 1. Tomba del Guerriero - 2. Tomba del Cacciatore - 3. Tomba della Pulcella - 4. Tomba del Fior di Loto - 5. Tomba delle Leonesse - 6. Tomba della Caccia e della Pesca 7. Tomba dei Giocolieri - 8. Tomba dei Festoni - 9. Tomba dei Caronti - 10. Tomba del Gorgoneion - 11. Tomba 7. Tomba dei Giocolieri - 8. Tomba dei Festoni - 9. Tomba dei Caronti - 10. Tomba del Gorgoneion - 11. Tomba clinio - 16. Tomba del Letto Funebre - 17. Tomba dei Baccanti - 18. Tomba dei Leopardi - 19. Tomba del Morto 20. Tomba del Maestro delle Olimpiadi - 21. Tomba del Tifone - 22. Tomba degli Scudi - 23. Tomba del Cardinale - 24. Tomba dell'Orco - 25. Tomba del Convegno - 26. Tomba del Topolino - 27. Tomba delle Pantere - 28. Tomba della Scrofa Nera - 29. Tomba Giustiniani - 30. Tomba Giglioli - 31. Tomba delle Bighe - 32. Tomba del Barone 33. Tomba del Frontoncino - 34. Tomba della Nave - 35. Tombe dei Tori - 36. Tomba dei Leoni Rossi - 37. Tomba 1. delle Olimpiadi - 38. Tomba del Gallo - 39. Iomba della Capanna - 40. Tomba degli Auguri.

2.
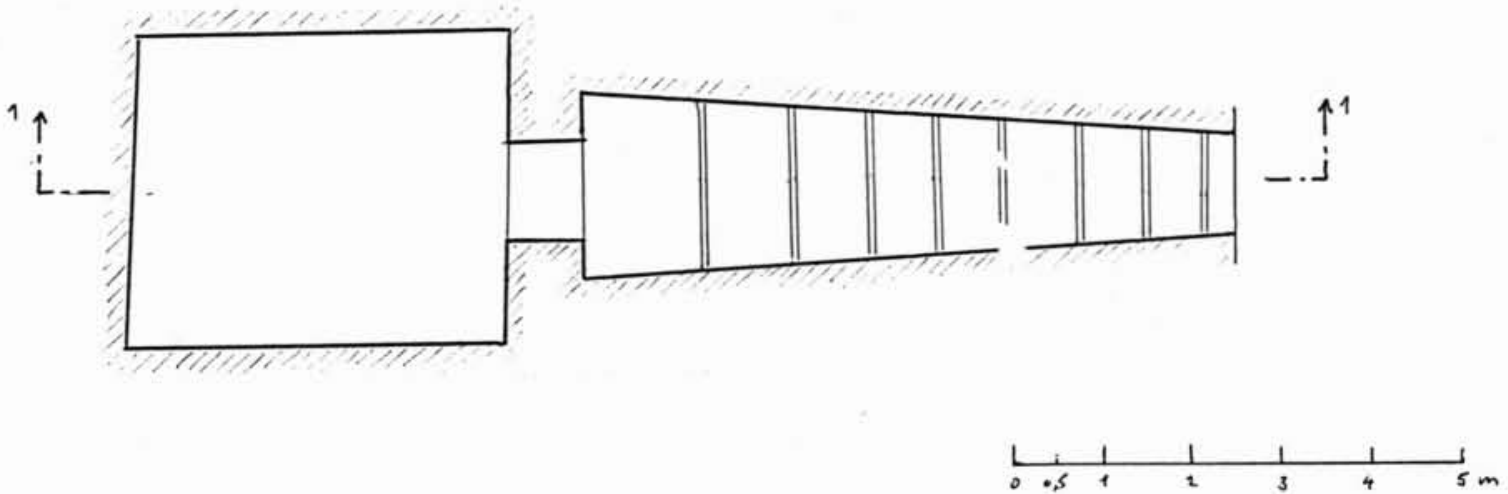

3.

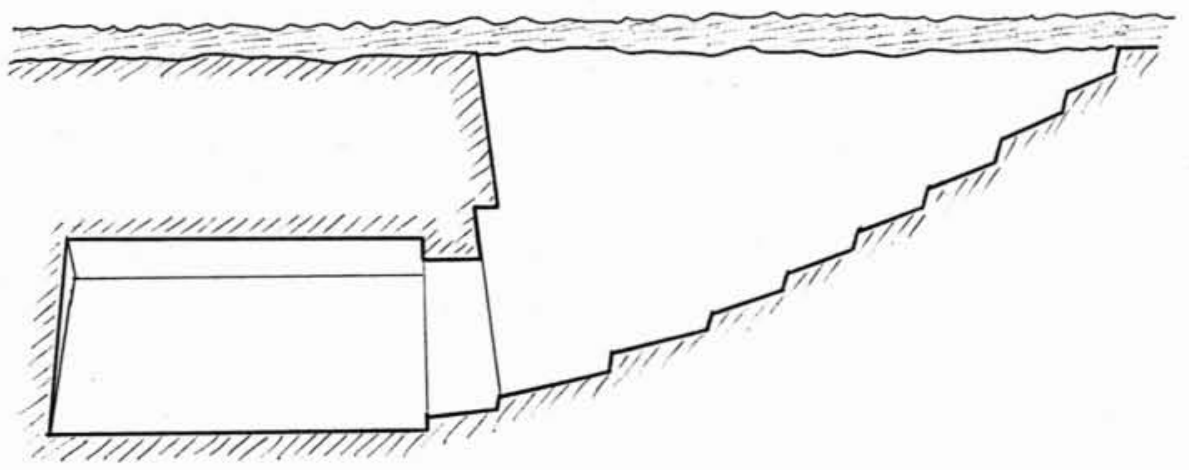

I. Localisation des tombes sur le plateau des Monterozzi (d'après [1 12$]$ ).

2. Plan de la Tombe du Navire (d'après [21]).

Face à l'entrée, la chambre sépulcrale. La scène maritime occupe l'extrêmité de la paroi latérale à gauche de l'entrée (vers 440 avant notre ère).

3. Coupe de la Tombe du Navire (d'après [21])

La coupe est effectuée suivant l'axe I-1 de la figure précédente. La tombe est creusée dans le tuf du plateau des Monterozzi (vers 440 avant notre ère). 
à la disposition des archéologues des moyens de prospection performants, adaptés à leurs problèmes [16].

Le premier consiste dans la détection d'anomalies dans la résistance électrique du sol, à partir de mesures effectuées en surface, suivant un réseau suffisamment serré pour permettre la mise en évidence de structures vides ou pleines, de nature différente, à une profondeur de quelques mètres $[1][21]$. Cette prospection est éventuellement assortie d'une recherche d'anomalies magnétiques du sol, au moyen d'un magnétomètre différentiel très sensible.

Ensuite, un forage de faible diamètre (quelques centimètres) [21], donc très peu dommageable aux structures, permet d'introduire un périscope imaginé par Nistri [31] [32] (sur le columen de la Tombe Cardarelli $\left(\mathrm{N}^{\circ} 809\right)$ on voit le trou de forage débouchant, par chance, juste au centre d'une rosace de la décoration [21]). Ce périscope permet l'observation directe, riche d'enseignements sur la forme de la tombe, sa décoration, son état de conservation, et la position de l'entrée, précieuse dans l'éventualité d'une fouille. Il était également possible d'y adjoindre un appareil photographique type Minox [31] [32], permettant d'obtenir des images de l'intérieur de la tombe.

Un dernier perfectionnement, dû à G. Parenti, consiste à déplacer verticalement le point de prise de vue d'une hauteur bien connue; la comparaison de deux images prises des deux points réalise alors une télémétrie assez précise [31] [32], permettant de relever le plan de la tombe. R.E. Linington [17] a mis en garde toutefois contre les erreurs qui peuvent résulter de cette télémétrie, erreurs qui, pour les tombes les plus grandes, peuvent atteindre plusieurs décimètres si le forage n'est pas bien vertical.

\subsection{Les premiers résultats des nouvelles méthodes de pros- pection}

Une première campagne de mesures, destinée seulement à évaluer les possibilités de la méthode, a eu lieu du $30 / \mathrm{s}$ au 2/6/1955. Puis, en 1956, du 15 au 20 septembre, une nouvelle campagne a permis de localiser aux Monterozzi une douzaine de tombes $[16][20]$.

A la suite de ce résultat extrêmement encourageant, une véritable campagne de détection a été entreprise pendant lc premier semestre 1958 ; elle a permis d'identifier 250 tombes, dont environ la moitié à chambre, les autres à fosse ou à inhumation. Les forages de contrôle ont été très laborieux à cause de la formation géologique du plateau des Monterozzi. C'est au cours de cette campagne que le périscope de Nistr a été utilisé pour la première fois, fournissant un millier de photographies environ. $99 \%$ des découvertes étaient saccagées et sans vrai intérêt archéologique, mais, vers la fin de la campagne, le 6 juillet, on découvrit la Tombe du Navire [16].

En 7 années d'exploitation de ces procédés de prospection, on a localisé plus de 6000 tombes, en majorité à chambre funéraire; parmi celles-ci, seulement une vingtaine ont une décoration peinte bien conservée. Rappelons que, avant cette époque, on connaissait en Etrurie une centaine de tombes peintes seulement, dont une partie importante était déjà sérieusement dégradée.

\subsection{La découverte de la tombe}

La Tombe du Navire a ainsi été découverte le 6/7/1958. Des images de sa décoration intérieure auraient aussitôt été transmises sur les chaines de télévision non seulement italiennes, mais américaines.

L'examen visuel révéla que la tombe avait été violée; le mobilier funéraire en avait disparu, à l'exception d'un sarcophage de pierre difficilement transportable. Près de l'entrée, un tas de terre témoignait d'une première effraction [20].

\section{Description de la tombe}

\section{I. Situation, voisinage}

La Tombe du Navire est située à Tarquinia, sur le plateau des Monterozzi, qui en recèle un grand nombre; elle se trouve dans la partie orientale de la nécropole (fig. I), et voisine en particulier avec les tombes des Taureaux, des Lions Rouges, des Olympiades et des Augures.

Elle est citée maintenant par plusieurs auteurs, aussi bien des historiens de marine comme Jean Rougé [35], que des étruscologues (Giannini [12], Moretti [21], Steingräber [36], etc.).

\subsection{Description de la tombe, dimensions, date}

La Tombe du Navire est décrite dans des publications antérieures de Mario Moretti [19] [20]; elle comporte une seule chambre funéraire, sensiblement rectangulaire, de $4 \mathrm{~m} 70 \mathrm{de}$ longueur et $3 \mathrm{~m} 45$ de largeur ( $[20]$ et $[2 \mathrm{I}]$ donnent des dimensions très légèrement différentes) (fig. 2,3 ). Le sol est à environ $3 \mathrm{~m}$ so sous le niveau du plateau à cet endroit. On y descend par un dromos à plan trapézoïdal, de $6 \mathrm{~m} 30$ de long, large de $1 \mathrm{~m}$ 10 et $1 \mathrm{~m} 85$, dont les parois sont inclinées à l'extrèmité, et comportant un escalier raide de 9 marches de largeurs variées (o m 56 à $1 \mathrm{~m}$ i 5 ). L'entrée de la chambre est une porte de $1 \mathrm{~m} 80$ de haut, large de $\mathrm{t} \mathrm{m} 10 \mathrm{en}$ bas et de $\mathrm{t} \mathrm{m}$ en haut.

Le plafond est à double pente, avec poutre centrale (columen) en saillie de $7 \mathrm{~cm}$ et large de $1 \mathrm{~m}$ IO; sa hauteur maximale est, au centre, de $2 \mathrm{~m}_{32}$; les deux pentes s'élèvent de $1 \mathrm{~m} 78$ à $2 \mathrm{~m} 39$.

La tombe est datée du milieu du $\mathrm{v}^{\mathrm{e}}$ siècle avant J.-C., probablement vers -440 ; cette datation est justifiée principalement par l'étude de la scène du banquet [12] [21].

\subsection{Mobilier funéraire}

La tombe a été précédemment violée; la dalle supérieure de fermeture de la porte avait été enlevée, et en partie brisée. Le mobilier funéraire en était absent. Cependant, à droite et au fond de la chambre, on remarque sur le sol quatre anfractuosités rectangulaires qui devaient recevoir les pieds d'un lit funèbre disparu.

Toujours au fond, mais à gauche, est resté un sarcophage en nenfro. Le couvercle, cassé en trois morceaux, a la forme d'un toit à double pente avec poutre centrale; il a une longueur de $2 \mathrm{~m}$ to et une largeur de $\circ \mathrm{m} 75$; l'épaisseur au centre atteint $\circ \mathrm{m} \mathrm{14}$. La caisse a ses parois lisses ; sa longueur est de $2 \mathrm{~m} 02$ extérieurement et de $1 \mathrm{~m} 86$ intérieurement; ses largeurs externe et interne sont respectivement de o $\mathrm{m} 70$ et o $\mathrm{m} 54$; elle est profonde de o $\mathrm{m} 34$; sa hauteur externe, qu'on ne peut mesurer car elle est encastrée dans le sol, est de l'ordre de o m so.

D'autre part, de la terre, provenant vraisemblablement de visites précédentes, avait été accumulée près de l'angle droit d'entrée. En la triant avec soin, on a retrouvé des fragments d'une Kylix attique à figures rouges, de poculum grossier de terre cuite sombre, d'un bol de bucchero grossier, et de divers disques de terre cuite, ainsi que de quelques autres pièces de céramique [20].

\subsection{Etat des peintures et mesures de sauvegarde}

Les parois de la chambre, et surtout celle de droite et celle d'entrée, ont beaucoup souffert de l'humidité, qui a imbibé le calcaire ; de son fait, l'enduit (intonaco) s'est gonflé, soulevé, 


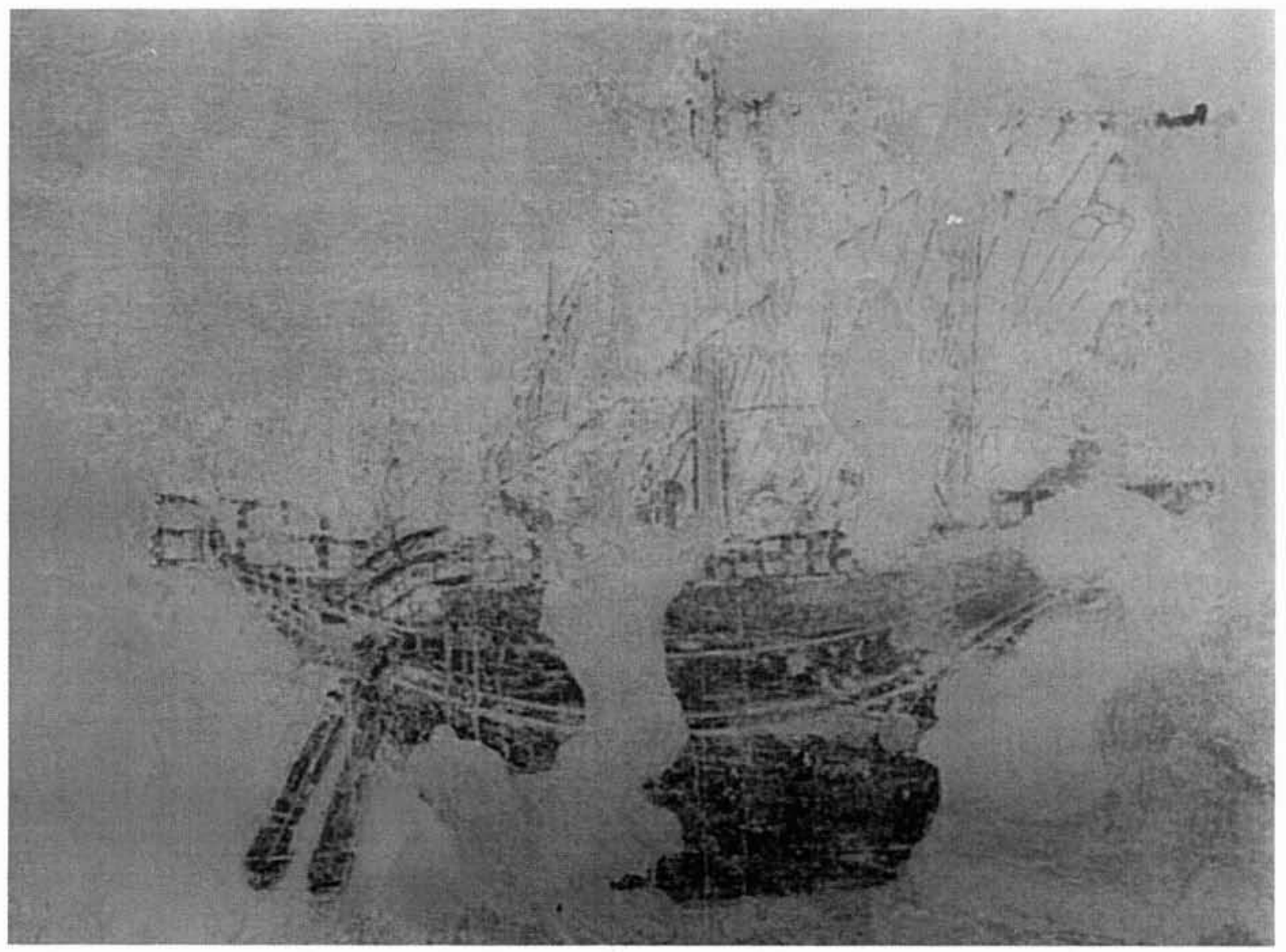

4. Scène maritime (d'après $[21]$ ).

Pour cette restitution sur palette graphique, l'image informatisée du navire a été dépouillée de tout ce qui ne concernait pas ce dernier. Copyright Pandore et Monique Bonnin.

5. Restitution du navire.

Cette restitution a été opérée sur palette graphique en tenant compte à la fois de l'image précédente et des travaux de restitution dus aux professeurs Sammarco et Zanelli [20]; les manques sont comblés par des aplats. Copyright Pandore et Monique Bonnin.

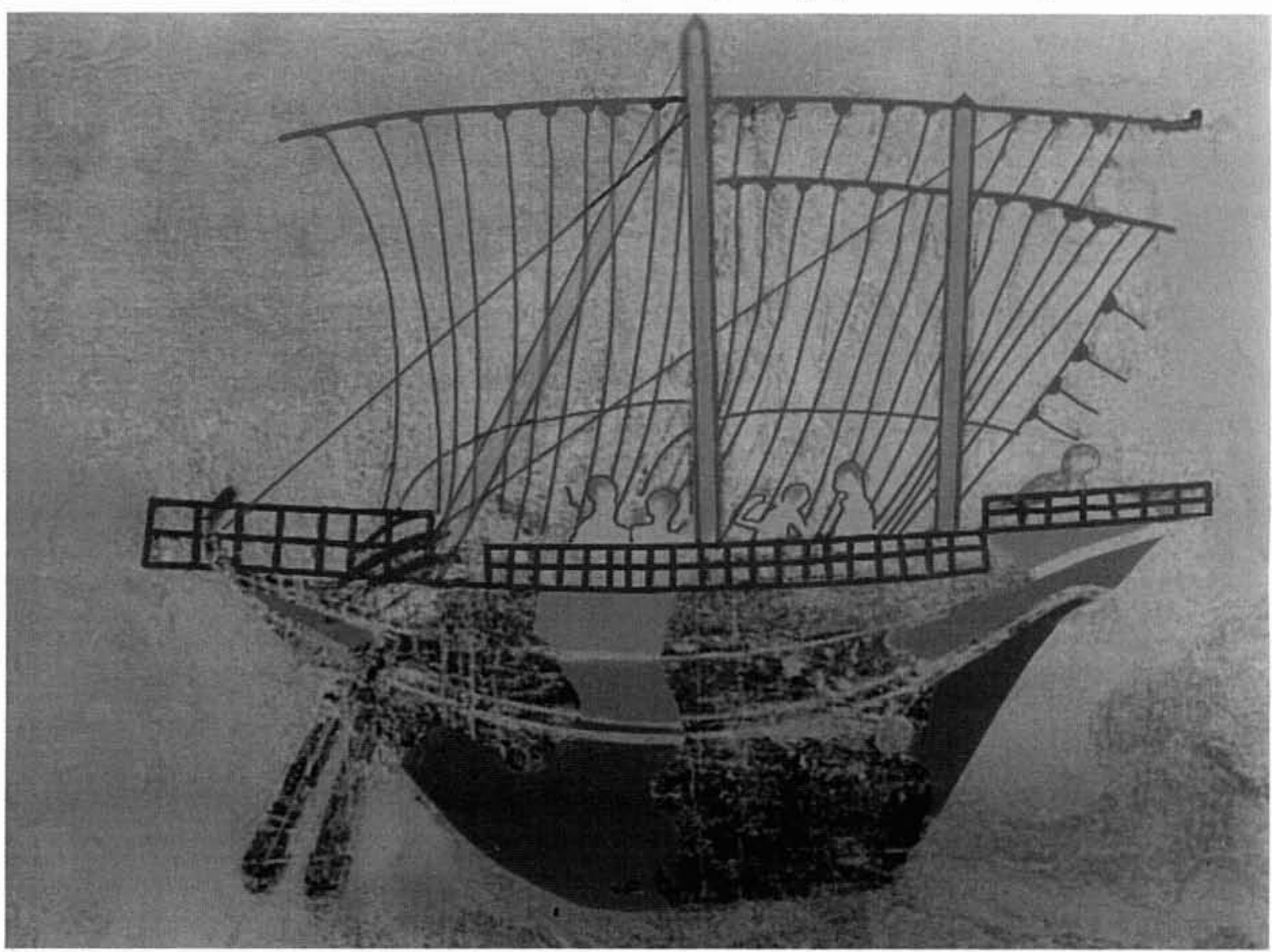


clivé, et par endroits détaché dans des zones étendues [19] [20|. D'autres zones étaient couvertes de salpêtre.

Dès sa découverte, l'état de conservation était préoccupant. Il fut décidé de procéder sans délai à la dépose des fresques, ce qui fut effectué par les spécialistes de l'Institut Central de Restauration; les résultats ont été surprenants.

On a détaché la fresque de chaque paroi d'un seul tenant, en la reportant sur une robuste toile de chanvre, fixée sur un nouveau type de toile métallique. Le procédé, imaginé par le Docteur Roberto Caritả, avait déjà été utilisé avec succès pour la tombe (voisine) des Olympiades.

\subsection{Description de la décoration}

Plafond. Les pentes du plafond sont décorées en damiers de - $\mathrm{m} 10$ de cóté, peints en brun rouge et réservés. Ce décor, parfois interprété comme l'envers des tuiles d'un toit, pourrait, comme dans d'autres tombes (tombe du Chasseur par exemple), indiquer la toiture d'une tente $[13]$. Le columen (poutre centrale) était jadis peint en rouge, et repose sur deux corbeaux ou consoles (monachi) de méme couleur, placés au milieu de petits frontons sculptés dont le décor a en grande partie disparu.

Murs. Une frise portant le décor principal, haute de $1 \mathrm{~m}$ à $1 \mathrm{~m} \mathrm{12}$, ornait toutes les parois. Cette frise est limitée en haut et en bas par deux séries semblables de 7 bandes horizontales polychromes, larges de $18 \mathrm{~cm}$, partiellement peintes et partiellement réservées. On trouve en partant du haut les couleurs suivantes : brun - réserve - rouge - réserve - brun - réserve rouge. Sous la série inférieure, le blanc du fond se continue jusqu'au sol, sur une trentaine de centimètres.

Conformément au schéma décoratif classique pour les tombes Tarquiniennes du $\mathrm{v}^{\mathrm{c}}$ siècle avant notre ère, la Tombe du Navire développe sur la paroi du fond, celle qui est le plus en valeur, la représentation d'un banquet ; celle-ci est encadréc sur les parois latérales, à gauche comme à droite, par celles de l'environnement de ce banquet : les musiciens et danseurs qui l'accompagnent, le kylikeion (que nous pourrions traduire approximativement par « vaissellier ") et sa riche exposition de vaisselle de luxe, bien conservée. Ce kylikeion est précédé, à gauche, d'une scène maritime essentiellement consacrée à la représentation - exceptionnelle dans les tombes étrusques d'un navire de commerce de grande taille, objet de notre intérêt ici, et dont on trouvera la description au $\$ 3.6$.

Les différentes scènes de la décoration sont rythmées plutôt que séparées par de légers arbustes, tracés en rouge. Une variété de vases précieux, richement décorés, accrochés au mur ou posés sur une table, témoigne de l'aisance du maitre de céans. Un joueur de cithare introduit le banquet à gauche, alors qu'un autre probable musicien le conclut sur la paroi de droite, vraisemblablement accompagné de danseurs.

Mis en valeur sur la paroi du fond, un ensemble de trois klinai (lits) supporte autant de couples de banqueteurs, richement vétus et parés, dans des attitudes volontairement diverses, servis par des esclaves nus [20]. Leurs chaussures sont restées au sol, devant des escabeaux bas aux pieds en pattes d'animaux. Un quatrième couple prolongeait la scène sur la paroi de droite, avant musiciens et danseurs : il n'en reste pratiquement rien. C'est l'étude de cette décoration qui a permis a M. Moretti [20] de dater la tombe de -440 environ.

\subsection{Description de la scène maritime}

La plus grande partie du mur de gauche, après une zone très lacunaire, est occupée par une scène maritime. Celle-ci est présentée en couleurs par M. Moretti dans les réfẻrences [20] et $[21]$.

Un traitement informatique sur «palette graphique " nous en a permis une présentation clarifiće. La figure $f$ montre le navire de commerce qui y figure, dépouillé des autres éléments du décor (barque annexe, rochers, etc...). Sur la figure s nous avons pu restituer l'apparence la plus probable du seul navire entier, les ajouts de restitution étant en aplats de mème teinte que les parties conservées de la fresque, mais s'en distinguant sans ambiguïté.

La scène maritime (figure s) fait apparaitre un grand navire de commerce, à coque ronde (cf. Homère), à proue et poupe relevées, portant deux mâts et leur gréement. Des câbles retiennent les voiles et augmentent leur résistance au vent. $\mathrm{La}$ coque est peinte en brun rouge (?) [20]. A la poupe, vue en perspective, une sorte d'échelle semble devoir servir au débarquement lorsque le navire est accosté. Un bastingage délimite le pont supérieur. A l'arrière, deux grands avirons servent de gouvernails. Au sommet du grand mát, une hune devait servir de poste de vigie.

La couleur bleu-vert de la mer a disparu presque totalement. A droite, un gros écueil est représenté de façon conventionnelle (cf. \$3.7), par une stratification de bandes ondulées colorées (cf. la Tombe des Taureaux et la Tombe de la Chasse et de la Pêche). Un homme, au-delà des rochers, semble observer la scène.

Mario Moretti $[20,26]$ donne une reconstruction du navire, due aux professeurs Renzo Sammarco et Antonio Zanelli (utilisée pour élaborer la figure 5 ), qui n'a pu que s'inspirer des connaissances de la construction navale et de la navigation dans cette région du monde et à cette époque.

Pour interpréter cette scène, il est bon d'avoir recours à ce que nous savons des navires de l'époque, en Etrurie et dans les pays avec lesquels elle commerçait, des conditions, et de ce qui a pu nous rester de ces navires antiques, sous forme d'épaves, ou dans l'iconographie, ou dans les textes, ainsi que des enseignements de ces informations sur leur construction (cf. $\S$ s).

\subsection{Les rochers: convention picturale}

Près de la proue du navire est figuré un gros écueil rocheux, traité de façon similaire à ceux des tombes des Taureaux et de la Chasse et de la Pèche. Ce rendu est plus conventionnel que réaliste: l'intonaco (enduit) est couvert de bandes de couleur (brun-rouge et violet) ondulées, qui peuvent faire penser par leur tracé aux stratifications de certaines roches, mais avec des couleurs inattendues $[20,23,25]$.

La parenté avec les deux autres tombes mentionnées cidessus est évidente à première vue. Dans l'espace, celle des Taureaux est toute proche, alors que la Tombe de la Chasse et de la Péche se trouve dans la zone occidentale de la nécropole; dans le temps, cette dernière est antérieure de 60 à 80 ans, celle des Taureaux un peu plus ancienne probablement $[12,22,28]$. Il est donc exclu que le peintre du Navire soit le mème; il a pu néanmoins connaitre les décorations précédentes, ou avoir la méme source d'inspiration pour ce détail du décor, assez peu répandu.

\section{Le navire de transport dans l'histoire maritime de l'Étrurie}

\subsection{La domination étrusque sur la mer Tyrrhénienne}

La qualification de Tyrrhénienne, c'est-à-dire d'étrusque, affectée à la mer qu'entourent Italic, Sicile, Sardaigne et Corse, n'était pas usurpée, du moins à l'époque de l'apogée étrusque ; les Etrusques y ont alors commercé librement, riches de leurs céréales et de leur fer, avec les Carthaginois (comme l'attestent les échanges avérés entre Sardaigne et Etrurie). Mème encore au $\mathrm{Vi}^{\mathrm{e}}$ siècle, les menaces grecques sur la Corse se sont semblet-il soldées, à Alalia, à l'avantage des Etrusques [s].

Leur extension vers le sud, en Campanie - dont la côte ne manquait pas d'installations grecques - ne pouvait se conce- 
voir sans une relative sécurité des transports maritimes [29] [35].

Par ailleurs, ils ont étendu leur commerce (parfois fait de piraterie) en Egée et en Méditerranée occidentale, de Lemnos à Malaga, et peut-être jusqu'à Madère, justifiant l'appellation de "thalassocratie étrusque ».

A Massalia, selon P. Pomey [3 2a], «la présence du commerce étrusque est bien attestée au - VI ${ }^{\mathrm{e}}$ siècle par plusieurs épaves, comme celle d'Antibes " - et d'ailleurs celle de Bon Porté [14a].

Pendant les siècles qui couvrent le deuxième quart du premier millénaire, il est donc certain que les Etrusques ont entretenu une flotte de commerce capable d'assurer leurs échanges, en position forte, et, pour lui garantir, une suffisante sécurité ; des bâtiments de combat ou des navires de charge équipés de manière à pouvoir se défendre (cf. $\S$ 5.1). Bien des auteurs s'intéressent beaucoup plus aux navires de combat qu'à ceux voués au seul transport des marchandises.

\subsection{Autour de la bataille de Cumes}

Présents en Mer Tyrrhénienne depuis le début du VIII ${ }^{\mathrm{e}}$ siècle (à Ischia), les colons en provenance de diverses cités grecques n'ont pas manqué de vouloir s'affranchir de la domination des Etrusques sur cette mer. Ceux-ci, alliés aux Carthaginois qui avaient le même intérêt à limiter l'expansion grecque, ont longtemps pu résister. La situation a changé très près du milieu du millénaire.

Selon M. Pallottino [30], dès la première décennie du $\mathrm{V}^{\mathrm{e}}$ siècle, les marins des îles Lipari auraient prêté la main à Denis de Phocée dans des actes de "piraterie" contre les Carthaginois et les Etrusques.

Entre 490 et 48 s, les Etrusques auraient lancé une expédition punitive contre les Lipariens, que ces derniers repoussèrent. Ce fut l'occasion d'un anathème de Delphes contre les Etrusques, objet d'une inscription gravée sur calcaire [30].

Peu d'années plus tard, entre 48 s et 480 , les Etrusques seraient revenus en force, conquérant Lipari, s'installant dans l'archipel, où ils se seraient livrés à des sacrifices humains. Une contre-offensive des Lipariens aurait libéré l'île, entre 480 et 475 , grâce à une mancuvre navale décrite par Pausanias (X, 11,3 ), peut-être avec l'aide de Syracuse, suivie d'un deuxième anathème de Delphes.

Avec la défaite navale des Etrusques à Cumes, c'en était fini de leur présence dans les Iles Eoliennes, et de leur implantation efficace en Campanie. Il est néanmoins probable, toujours selon M. Pallottino [30], qu'ils intervinrent aux flancs des Athéniens contre Syracuse en $4{ }^{1} 4-413$, sous le commandement de Velthur Spurinna.

\subsection{Survivance du commerce maritime étrusque}

En dépit de ses revers, et de la diminution de sa domination géographique et économique, l'Etrurie a continué à commercer et à vivre activement pendant plusieurs siècles, ce qui implique le maintien d'une flotte, et en particulier d'une flotte de commerce. On ne doit pas s'étonner, par conséquent, de voir figurer, au milieu du $\mathrm{v}^{\mathrm{e}}$ siècle, siècle qui voit s'amorcer le déclin de l'Etrurie, une représentation d'un important navire de commerce.

\section{Connaissance des navires antiques}

L'interprétation de toute scène maritime, et en particulier la reconstruction de la fresque de Tarquinia représentant un navire étrusque, s'appuie sur des sources de différentes natures qui nous renseignent sur l'aspect, la construction et l'utilisa- tion de ces navires. Ces sources sont les textes anciens, les vestiges de navires, et l'iconographie.

Comme les navires ont beaucoup circulé, on ne doit pas s'étonner de trouver des similitudes importantes entre ceux de pays qui ont multiplié leurs échanges, qu'il s'agisse de leur mode de construction, de leur gréement, de leurs méthodes de navigation [s] [29], donc en particulier entre les péninsules italique et hellénique et la côte massaliète.

\subsection{Les textes anciens}

C'est là que nous trouvons les sources sinon les plus riches, tout au moins les plus abondantes, rendant illusoire d'en faire un examen exhaustif. Strabon, Tite-Live, Pausanias par exemple nous renseignent à de nombreuses reprises. Citons seulement ici un texte qui permet d'illustrer la pérennité des types de navires. Homère, dans l'Odyssée, mentionne très souvent des "nefs longues" et des "nefs rondes". Situées au XII1" siècle avant notre ère, ces différences traduisent un fait plusieurs fois millénaire. Les nefs longues, rapides et seules utilisées au combat, sont couramment 6 à 8 fois plus longues que larges; elles peuvent être mues à l'aviron ou à la voile, ou même conjointement par les deux moyens. Les nefs rondes, mieux adaptées au transport des charges, sont seulement 3 à 4 fois plus longues que larges, et vouées au commerce. Elles ne peuvent guère être propulsées qu'à la voile, ce qui en limite les conditions de navigation. Entre ces deux types de proportions, un vide presque complet.

Un auteur, Sergio Paglieri [27], considère un troisième type, sous le nom de " navire étrusco-italique ». Il s'agit en fait d'une nef ronde, mais munie d'un éperon comme beaucoup de nefs longues - et que nous pouvons donc rattacher au deuxième des types précédents. Cette conception correspond à la nécessité de permettre aux navires de charge de se défendre contre les pirates - souci que l'on retrouve au Moyen Age encore dans la république maritime de Venise. Selon cet auteur, elle aurait déjà été présente dans la Crète Minoenne, mais sans qu'il soit possible de déterminer de façon sûre si elle a été transmise aux peuples italiques ou si elle en provenait. Pline la décrit en ces termes :

"Rostra addidit Pisaeus Tirrenus »

affirmant ainsi que c'est un Etrusque originaire de Pise qui aurait eu l'idée d'ajouter un éperon ou rostre aux navires de commerce. En fait, R. Rebuffat [33] montre que cette arme était inconnue jusqu'au $\mathrm{IX}^{e}$ siècle avant J.-C., mais qu'elle apparaît à peu près sûrement au vim ${ }^{\mathrm{e}}$. Cet éperon a été tantôt immergé, beaucoup plus nocif et faisant corps avec la structure du navire, tantôt aérien, ayant l'air seulement surajouté.

L'intérêt de la remarque concernant les deux types principaux est dans leur pérennité, tous deux bien antérieurs au ${ }_{1}^{\text {er }}$ millénaire avant notre ère, et toujours existants, dans leur évolution, avec des variantes certes et des adaptations, au $+\mathrm{XVII}^{\mathrm{e}}$ siècle, dans la Venise des Doges comme dans toute l'Europe.

Bien d'autres textes décrivent la construction, la mancuvre des navires. Certains n'éclairent pas le sujet, ou sont devenus compréhensibles lorsque l'on a pu étudier des épaves. D'autres ne nous aident pas encore à comprendre unanimement l'économie des trières ou trirèmes.

\subsection{Vestiges de navires}

Les conditions de conservation du bois nous privent malheureusement de bien des reliques. Il faudrait soit une sécheresse extrême, soit une constante humidité. Seul le climat de l'Egypte a permis de conserver pendant des millénaires des bateaux, mais trop éloignés de nous pour être retenus ici.

En revanche, la mer conserve très longtemps les épaves de ceux auxquels elle a été funeste. Depuis quelques décennies la 


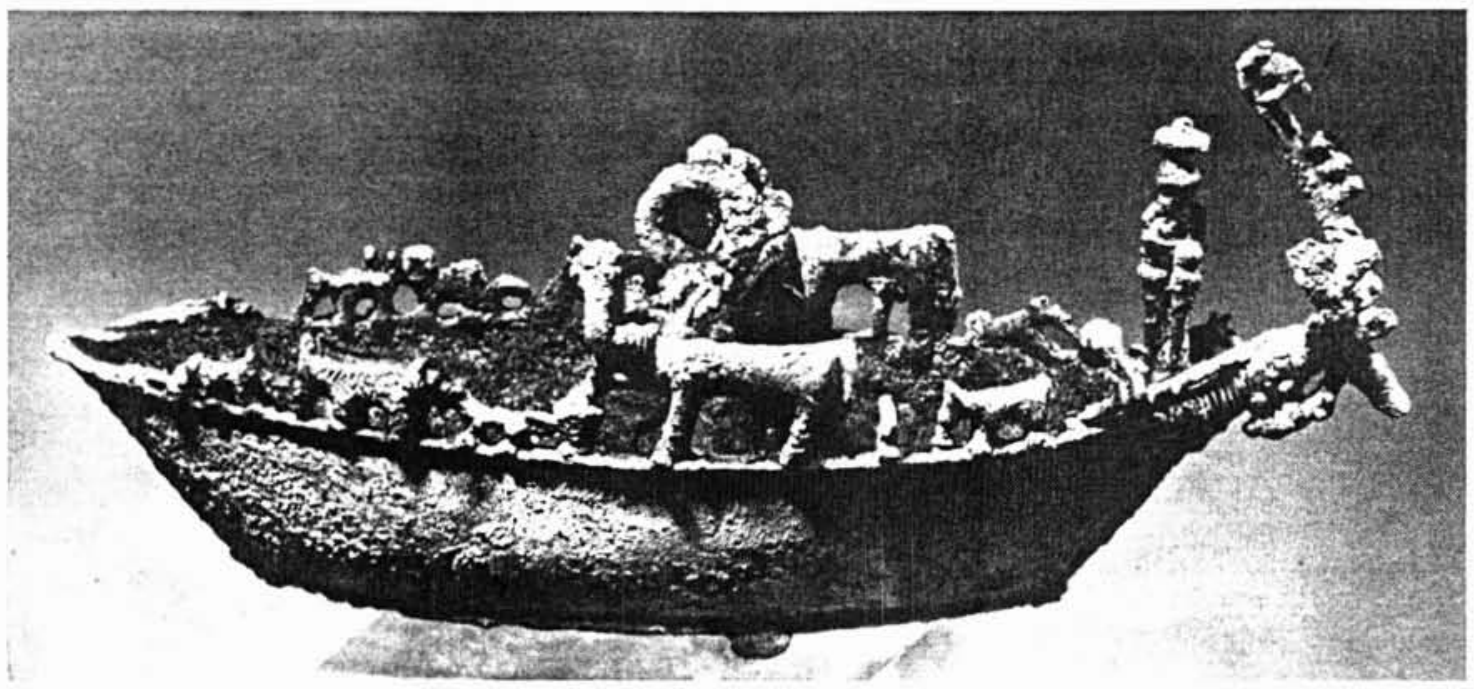

6. Modele de barque étrusque (Musée archéologique de Florence; d'après [2]).

Cette barque, peuplée d'animaux, a été retrouvée à Vetulonia dans la Tomba del Duce ; elle est datée de la fin du VII ${ }^{e}$ siècle avant notre ère.

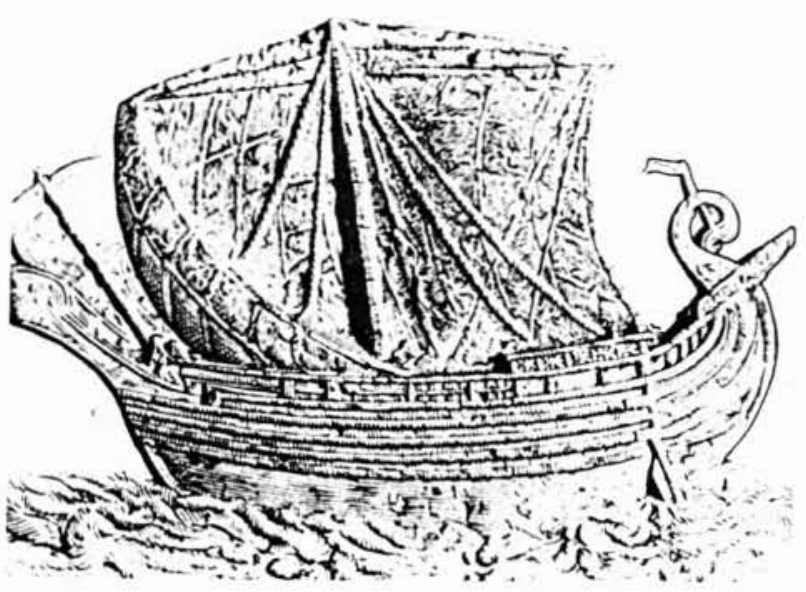

fouille des épaves, détaillée, a permis d'augmenter considérablement nos connaissances des navires antiques.

La barque étrusque retrouvée à Spina, et conservée en assez bon état au musée archéologique de Ferrare, est trop petite et trop différente d'un navire de charge pour nous intéresser ici.

P. Johnstone [15], M. Gras [14] mentionnent deux épaves étrusques du - $\mathrm{vi}^{e}$ siècle, à Antibes et à Bon Porté (SaintTropez). La première, étudiée par Pruvot [32b], Cl. AlboreLivadie [1a], B. Bouloumié [sa], n'a laissé que sa cargaison, en majeure partie étrusque; la seconde, étudiée par L. Long et F. Richez [17a], était chargée surtout d'amphores étrusques. Toutes deux attestent l'exportation de vin étrusque vers la région de Massalia, la production massaliète de vin étant à l'époque insuffisante.

Nous pourrons en revanche comparer notre navire à l'épave de Kyrenia [15] navire de commerce probablement chypriote ou grec, probablement plus petit que le nôtre, mais qui a l'avantage d'avoir été daté par le carbone 14 et situé au début du $-\mathrm{IV}^{e}$ siècle, soit seulement quelques décennies après la fresque de Tarquinia.
7. Bas-relief d'un natire de Tartessos (Liban) (d'après [11])

Ce navire est tout à fait semblable aux navires celtiques qui attaquèrent en - 55 Jules César, dont nous en tenons la description: coque ronde, aviron de gouverne, mât haubané, voile à renforts horizontaux et verticaux.

\subsection{Iconographie du navire}

L'iconographie des navires comprend toutes leurs représentations, qu'il s'agisse de modèles réduits, de sculptures ou bas-reliefs, d'urnes funéraires, de stèles gravées, de peintures murales ou de fresques, de peintures sur vases, d'incisions décoratives sur pièces métalliques diverses (miroirs, cistes...), de monnaies frappées ou moulées. Dans le cas des peintures sur vases, la courbure du support incite l'artiste à des déformations dans le dessin, qui rendent délicats certains aspects de son interprétation. Pour les monnaies, une schématisation due à l'exiguité du support la rend également souvent hasardeuse.

Les modèles réduits de bateaux, assez fréquents dans l'Egypte et dans la Crète antiques, sont rarissimes en Etrurie ; R. Bianchi-Bandinelli et A. Giuliano [2] en situent un, en bronze, au VII ${ }^{c}$ siècle, à Vetulonia; mais il s'agit seulement d'une petite barque (fig. 6). S. Paglieri [27] cite plusieurs urnes cinéraires en albâtre, du - $\mathrm{II}^{\mathrm{c}}$ siècle, à Volterra, représentant divers navires. D'époque hellénistique également provient le grand relief de l'acropole de Lindos, sculpté dans le rocher en place, qui montre la proue d'une nef avec son rostre. Un autre bas-relief, trouvé au Liban, (fig. 7) montre une nef originaire de Tartessos, apparentée aux navires celtes de l'époque de la conquête romaine. Des monnaies de Volterra figurent également des navires. 


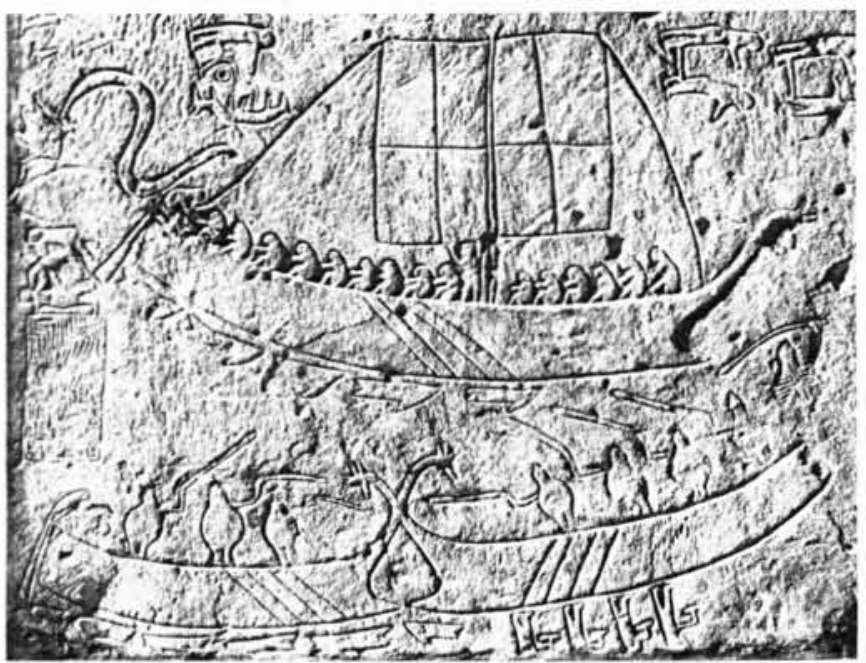

9.

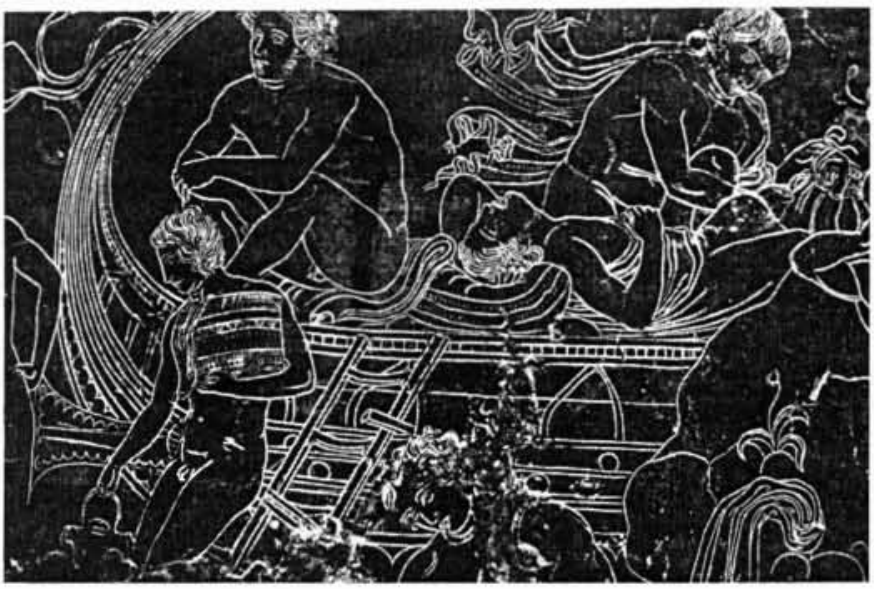

8. Stèle de Novilara (Musée Oliveriani, Pesaro; d’après [2]).

Certaines scènes de cette stèle, trouvée dans la nécropole de Novilara, près de Pesaro, illustrent la navigation et une bataille navale $\left(\mathrm{VI}^{e}\right.$ siècle avant notre ère).

9. Ciste Ficorini (Rome: Musée de la Villa Giula - d’après [37]).

Une des scènes gravées sur cette ciste en bronze trouvée à Praeneste (Palestrina) représente le navire des Argonautes, l'Argo, construit et armé à l'instigation d'Athéna (milieu du IV $\mathrm{V}^{\mathrm{e}}$ siècle avant notre ère).

Un certain nombre d'objets portent des représentations de navires gravées ou incisées. Au musée Oliveriani de Pesaro, on voit trois navires sur la stèle de Novilara (Piceno) (fig. 8), illustrant maladroitement leur gréement [3] du vII ${ }^{e}$ siècle [2] un autre, à la même époque, apparaît sur une situle en ivoire. A la fin du $\mathrm{vu}^{\mathrm{c}}$, un navire figure sur une autre stèle, à Siponte (Pouilles). Plus tardivement, la ciste Ficorini, du milieu du - $\mathrm{IV}^{\mathrm{e}}$ siècle (musée de la Villa Giulia) (fig. g) montre une scène incisée située à bord [37] du navire Argo. Enfin, $R$. Rebuffat [33] nous montre un navire incisé sur un vase de Véies du VIII ${ }^{\circ}$ siècle avant J.-C. En revanche, nous n'en avons pas trouvé de représentation sur des dos de miroirs étrusques.

En peinture pariétale, où la planéité du support évite les erreurs d'interprétation qu'occasionne parfois la courbure dans le cas de la poterie, seule existe encore, à part quelques petites barques, en dehors de notre grande fresque, la trop fragmentaire représentation de la Tombe du Navire de Cerveteri (fig. 10 ). Cette tombe, situće dans le tumulus éponyme, a été présentée pour la première fois par Raniero Menganelli [18]; P. Giannini [12] la date de la fin du $\mathrm{VII}^{e}$ siècle et M. Moretti [24] d'environ - 600. Les restes peints sont extrêmement rudimentaires et rarement reproduits. D'autre part, d'après St. Steingräber $\left[{ }_{3} 6\right]$, une autre tombe de Tarquinia aurait comporté une représentation de navire, maintenant complètement disparue.

Pourtant, ce type de représentation est déjà très ancien : en témoignent les fresques d'Akrotiri (île de Thera, en mer Egée) antérieures d'environ un millénaire, qui montrent plusieurs «nefs longues".

Reste la peinture des vases, beaucoup plus souvent grecque qu'étrusque. On peut être surpris au premier abord par le fait que des pays aussi maritimes que la Grèce et l'Etrurie, commerçant entre eux et avec leurs voisins essentiellement par mer, aient laissé dans leur iconographie sur céramique une place aussi réduite à leurs navires, et en particulier à leurs flottes de commerce. Une vue d'ensemble en est offerte par le "Répertoire des vases grecs et étrusques " établi par Salomon Reinach dans les dernières années du XIX ${ }^{e}$ siècle [34] : sur des milliers de scènes peintes sur vases, une demi-douzaine seulement (toutes époques confondues) montrent des figurations de navires; encore s'agit-il le plus souvent de navires de guerre $[5,7,37]$

Des inventaires partiels, plus récents, apportent toutefois une moisson supplémentaire. Dans celui de J. Boardman "Athenian black figure vases" [4] on trouve cinq représentations, échelonnées au long du $-\mathrm{Vl}^{e}$ siècle, celui qui précède notre fresque. D'autres travaux, comme l'article de R.T. Williams « Early Greek Ships of two levels " [38] en montrent près d'une dizaine, pour certains confondus avec ceux d'autres références.

Citons quelques-unes de ces représentations, parmi les plus utiles à notre étude :

- le Vase François (décoration du col) (Musée de Florence); - le dinos d'Exechias (Villa Giulia sos99);

- la coupe à figures noires du British Museum (B 436);

- l'amphore à figures noires du British Museum (B s08);

- l'amphore du Musée de Tarquinia (décoration du col) $\mathrm{N}^{\circ} 678$;

- la fameuse oenoché représentant Ulysse et les sirènes;

- le vase du Musée du Louvre A 517 ;

- le cratère de Bruxelles (ex Louvre A 531);

- le cratère de Cerveteri du - vi ${ }^{\mathrm{e}}$ siècle (Louvre);

- le cratère de Buchner, de la fin du viII ${ }^{\mathrm{e}}$, trouvé à Lacco

Lameno (île d'Ischia) (fig. II);

- le cratère de Cerveteri du milieu de - vil ${ }^{e}$ signé par Aristonothos (Rome, Palais des Conservateurs) : un navire de guerre et un navire de commerce se préparent probablement au combat (fig. 12).

L'ensemble de cette iconographie nous a servi pour interpréter le navire représenté dans la tombe de Tarquinia. 
6. Le navire de Tarquinia dans le contexte maritime du premier millénaire

A la lumière de ces différentes sources, nous pouvons maintenant étudier le navire peint sur la paroi gauche de la tombe. On pourra le considérer comme représentatif des navires de commerce étrusques, sinon grecs. En effet, l'insuffisance des informations nous semble interdire de songer à mettre sur pied une typologie même rudimentaire des navires suivant leur pays d'appartenance. Nous examinerons dans ce qui suit le navire, de la quille au sommet des mâts, y compris donc les parties non représentées sur la fresque de la tombe.

\section{I. Quille}

Nous savons par les textes que les Grecs enviaient aux Etrusques leurs forêts plus riches en beaux arbres bien droits, qui leur permettaient d'exécuter la quille des navires en une seule pièce. A défaut, ils étaient obligés de fabriquer, à partir d'une certaine longueur, une quille composite, en assujettissant solidement les madriers au moyen de l'assemblage encore connu aujourd'hui sous le nom de "trait de Jupiter" (sic) (fig. 13). Toutefois, cette remarque doit concerner essentiellement les nefs longues de combat, construites sur une longue quille droite, et non ce navire de commerce aux fonds extrêmement galbés.

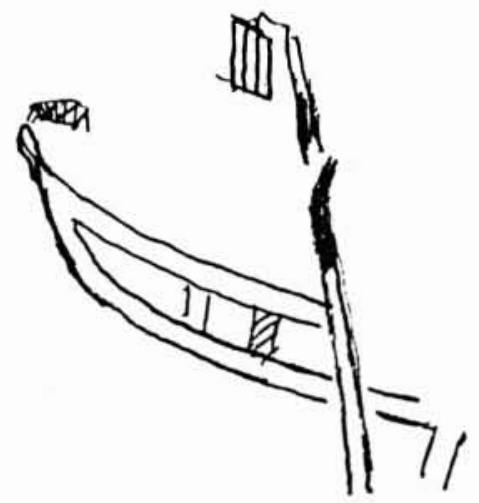

10.

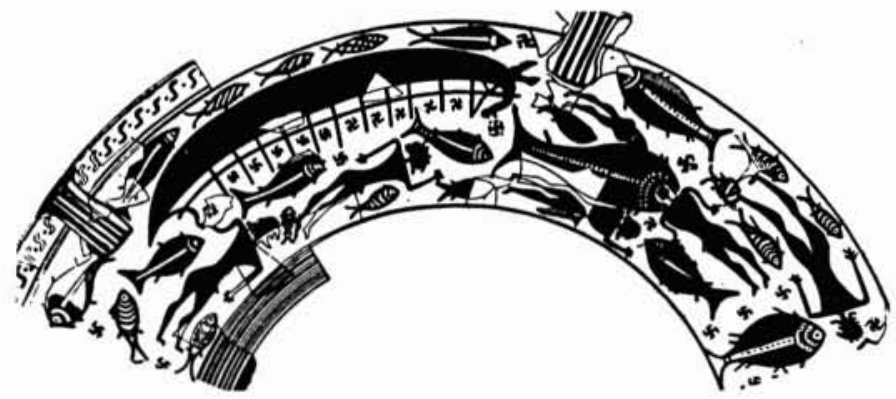

11.
12.

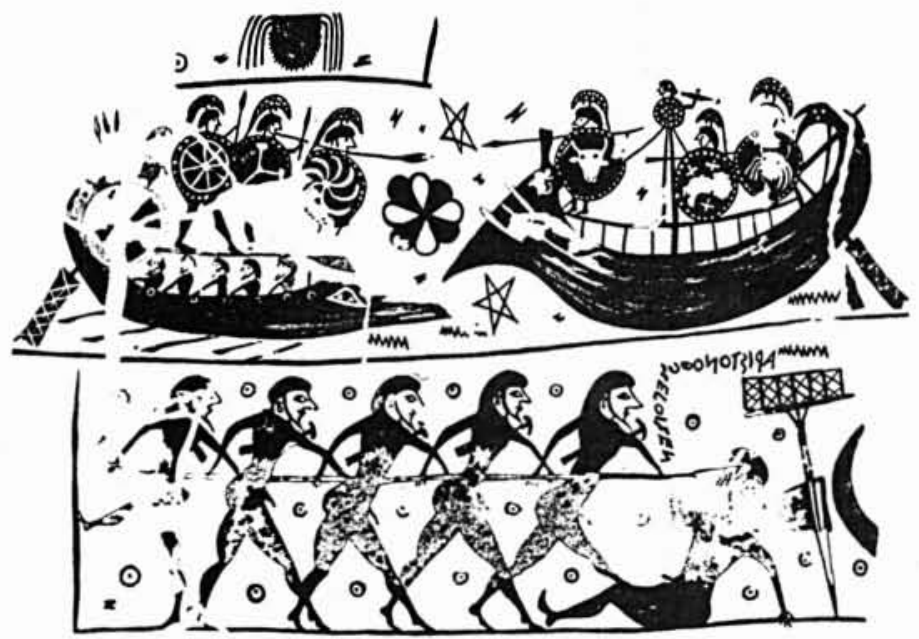

a

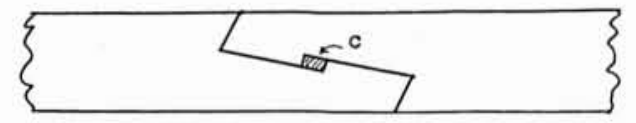

b

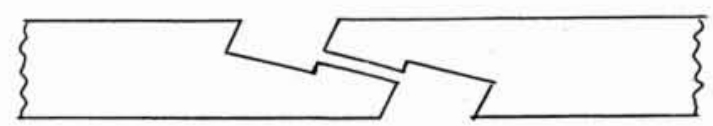

13.

IO. Tombe du navire à Caere (Cerveteri) (d'après [18]).

Cette tombe a donné son nom au grand tumulus de la Banditaccia (Cerveteri) qui abrite cinq groupes sépuleraux. Sur la paroi du fond de celle-ci restent des traces de peinture représentant un navire avec un mât incliné et une voile de couleur rouge.

I I. Cratère de Lacco Lameno (Antiquarium d'Ischia - d'après [2]).

Sur le dessin développé de ce cratère de la fin du $\mathrm{VIII}^{\mathrm{e}}$ siècle est représentée une scène de naufrage. Le navire est chaviré, et les marins noyés flottent entre deux eaux parmi les poissons, dont l'un commence à les dévorer.

I 2. Cratère d'Aristonotbos (Rome, Palazzo dei Conservatori - d'après [2])

Sur ce cratère trouvé à Cacre (Cerveteri) et signé par Aristonothos, le plus ancien des peintres grecs établis en Etrurie, est représentée une bataille navale; c'est en fait l'attaque d'une nef ronde de commerce (à droite) par une nef longue munie d'un éperon, à gauche (vers 650 avant notre ère).

13. Assemblage dit en "Trait de Jupiter"

a: les deux pièces assemblées; $\mathrm{c}$ : cale de blocage ;

b: les deux pièces séparées. 


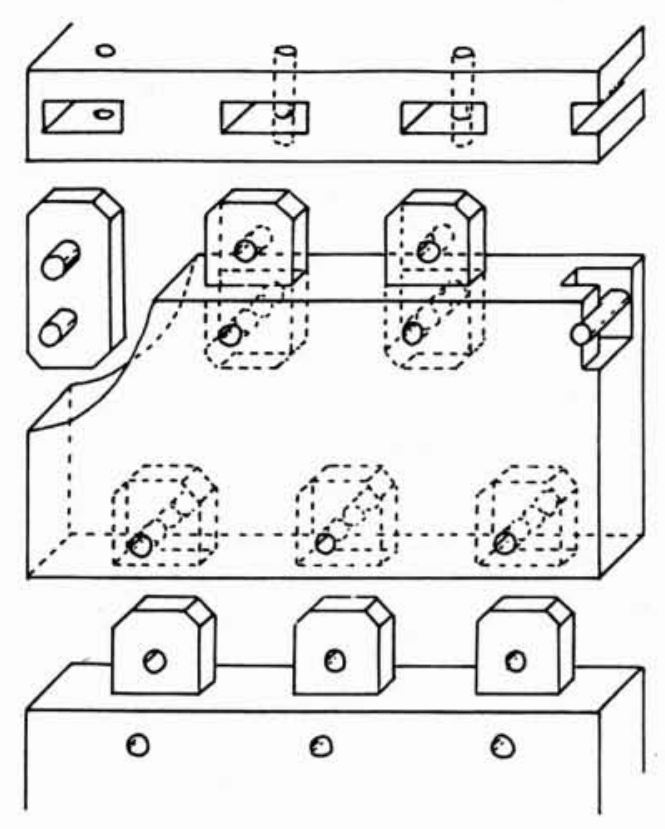

14. Assemblage du bordé d'un navire antique (d'après [15]). Les planches du bordé sont entaillées de séries de mortaises dans lesquelles s'engagent des tenons maintenus par des chevilles. $\mathrm{Ce}$ schéma a été relevé sur les restes du navire de Kyrenia (Chypre), du IV ${ }^{e}$ siècle avant notre ère.

\subsection{Coque}

C'est essentiellement l'étude des épaves qui nous renseigne sur la construction des coques de navires. Celle de Kyrenia étudiée par Johnstone $[15]$ a été datée par le carbone 14 entre -430 et -345 ; elle est donc très proche du navire de Tarquinia.

On sait maintenant que, à cette époque, les planches constituant le bordé étaient assemblées avant que l'on ne constitue le squelette fait de membrures, à l'inverse de la construction des coques de bois récentes.

Le procédé d'assemblage de ces planches est illustré par la figure 14. Des tenons en bois pénètrent dans des mortaises pratiquées dans le bordé, et y sont maintenues par des chevilles. On retrouve ce procédé sur d'autres coques d'époques différentes (par exemple celle du navire de la Bourse à Marseille). Sa découverte sur des épaves explique enfin des textes anciens longtemps incompris. Déjà Homère décrit dans l'Odyssée la construction d'une coque par Ulysse - en termes restés obscurs jusqu'à récemment, et que cette découverte éclaire; Ovide, dans les Métamorphoses, décrit un naufrage dramatique en mentionnant la ruine des "cunei ", c'est-à-dire des "coins", qui sont les tenons ci-dessus. Ces deux témoignages illustrent la pérennité du procédé de construction.

Toutefois, l'épave étrusque de Bon Porté, au siècle précédent [17a], atteste encore "l'assemblage du bordé par ligatures, sans l'aide de clous ni de chevilles ». Ce procédé, qui aurait pu être utilisé pour le navire de la tombe de Tarquinia, était déjà pratiqué par les Egyptiens deux millénaires plus tôt, même pour des navires plus importants.

\subsection{Gouvernes}

La fresque de Tarquinia montre très clairement deux longs avirons de gouverne à l'arrière du navire, un sur chaque bord. Cet équipement était quasiment universel sur les navires antiques de quelque importance, même en Egypte; il se poursuivra longtemps, ne devant être supplanté par l'invention du gouvernail d'étambot qu'au Moyen Age [10].

\subsection{Echelles}

A l'arrière du navire, M. Moretti [20] signale " une charpente en forme d'échelle qui doit être un agrès utilisé pendant que le navire est accosté au rivage ". De telles échelles d'accès sont visibles sur d'autres figurations antiques de navires - en particulier sur la coupe B 346 du British Museum, ainsi que sur plusieurs vases à figures noires $\mathrm{du}-\mathrm{VI}^{\mathrm{e}}$ siècle cités par J. Boardman [4].

\subsection{Gréement}

Le gréement du navire a été bien reconstitué par les professeurs Renzo Sammarco et Antonio Zanelli [20, 26] (fig. 5). Il est inévitable que cette reconstitution se soit inspirée des connaissances maritimes et en particulier iconographiques des navires de commerce de cette époque - essentiellement des navires grecs, ce qui doit dans le principe interdire ensuite toute comparaison entre les navires grecs et étrusques.

Le navire porte deux mâts, ce qui indique un tonnage relativement élevé. D'après St. Streingräber [36], c'est la première représentation connue d'un navire antique à deux mâts. La voilure est du type carré, les voiles étant soutenues par une vergue horizontale supérieure, gréement classique à l'époque. Mario Moretti [20] souligne les câbles de renforcement de la voile, partant de nœuds sensiblement équidistants sur la vergue. Ces renforts étaient fréquents dans l'antiquité; on les rencontre aussi combinés à des renforts horizontaux, faits de câbles ou même de lanières de cuir, formant avec les précédents une sorte de quadrillage de résistance.

On peut voir un tel réseau de renforts sur une nef de Tartessos représentée sur une stèle au Liban [i I ] César [6] y aurait reconnu les caractéristiques de navires celtes (Vénètes ?) qui, vers - 55 , auraient avec succès attaqué la flotte romaine (il avait fallu attendre l'absence de vent pour permettre aux romains, équipés d'avirons, de les vaincre isolément au combat corps à corps).

Aux nœuds du quadrillage ci-dessus mentionné se situaient soit des trous dénommés " œils de pie", soit des anneaux. J. Rougé $[35]$ en discute l'utilité, et montre qu'ils servaient, au moyen de cargues, à diminuer la surface de voilure. De tels anneaux de bronze ont été retrouvés en grand nombre dans certaines épaves.

L'examen des parties encore nettes sur la fresque de Tarquinia montre l'absence de renforts horizontaux de la voilure, tout comme celle de cargues multiples utilisées commodément pour «prendre des ris». Il s'agit donc ici de simples renforts de la toile de lin.

\subsection{Hune}

Au sommet de l'un des mâts, on entrevoit les restes d'une masse sombre que $\mathrm{M}$. Moretti [20] considère comme une hune. De telles hunes existaient sur les navires antiques; on en voit sur diverses représentations, comme la situle d'ivoire de Chiusi, et peut-être au sommet du mât incliné visible sur les maigres restes de la "Tombe della Nave » à Cerveteri [1 8]. De ce poste une vigie pouvait apercevoir d'autres navires (amis ou pirates) guetter les côtes, et mieux détecter et signaler les récifs dans les zones de danger pour la navigation. 


\subsection{Cargaison}

On peut légitimement s'interroger sur le type de cargaison que devait transporter ce gros navire. Même si M. Cristofani [9] le pense affecté à des fonctions plus honorifiques qu'utilitaires, il nous paraît logique de nous questionner sur le type de marchandises qu'il pouvait exporter ou importer.

Dans toutes les marines de commerce, les navires de charge les plus importants ne sont pas affectés au transport des objets de valeur marchande élevée, qui, s'ils sont présents, ne représentent qu'une faible partie de la cargaison.

Il nous parait donc exclu que, au $-\mathrm{v}^{e}$ siècle, un tel navire ait été construit essentiellement pour l'exportation d'objets en fer, richesse de l'Etrurie à son apogée - ni pour l'importation de poteries grecques de valeur. Nous serions tenté plutôt de le voir assurer le transport de ce que nous appelons aujourd'hui "les pondéreux", matières relativement lourdes et encombrantes eu égard à leur valeur. Il pourrait en particulier s'agir de produits agricoles, céréales, vins de consommation courante, huile, etc... Mais, bien súr, en l'absence de textes, il ne peut s'agir que d'hypothèses, qui pourraient se trouver contredites par d'éventuelles autres découvertes.

\section{Conclusion : interprétation de la scène du navire dans la tombe}

La scène du navire est un reste unique dans le répertoire connu de la fresque étrusque - si l'on fait abstraction de la tombe du mème nom à Cerveteri, d'où un modique fragment nous est revenu. Ailleurs on trouvera au maximum de petites embarcations, de péche ou de service, comme celles qui semblent voisiner ici avec ce gros navire de commerce.

Nous pouvons légitimement nous interroger sur le choix de ce sujet, vraisemblablement imposé par le commanditaire à un peintre dont on a vu par ailleurs la haute qualité dans le rendu de scènes plus courantes [20]. A-t-il voulu, dans un but apotropaïque, conjurer le danger, pour ses biens, son navire, sa personne mème, dans une démarche en harmonie avec le caractère étrusque? ou bien, rendre hommage, par un digne ex-voto, aux divinités de la mer (mais elles sont apparemment absentes de la scène...), pour ses succès, sa richesse acquise dans le commerce maritime? ou, plus simplement montrer que le propriétaire et commanditaire de la tombe, commandant du navire ou plus probablement son armateur, avait dû sa fortune à l'habileté de la navigation et de l'affrêtement ?

Pour Mauro Cristofani [9], ce navire serait un "vaisseau d'honneur ". La qualification, imprécise, n'est présente chez aucun autre commentateur de la tombe; lui-même n'en donne aucune justification. Elle permettrait d'échafauder des hypothèses incontrólables sur une quelconque "parade" nautique, dans laquelle le personnage honoré ne serait pas nécessairement lié aux choses de la mer...

Ce que nous savons des Etrusques nous fait douter d'une représentation dictée par des motifs purement profanes méme si ceux-ci demeurent présents. Nous sommes dans une tombe, à la porte de l'au-delà, chez un peuple religieux, dépourvu de respect humain. Si rien ne permet d'affirmer dans cette représentation - le navire arrivé heureusement à bon port - la conjuration du péril marin, la simple figuration du navire au port, en bon état, est en elle-même un ex-voto. Quoi qu'il en soit, une telle scène serait sans objet dans une famille qui ne serait pas attachée à la mer : tout nous pousse à penser que le chef de cette famille, qui en a fait décorer la tombe par un artiste de talent, était vraisemblablement un riche armateur.

\section{Bibliographie}

[1] A. Aspinal. \& K. PiCKARD. - A direct-reading earth resistance meter. Prospezioni archeologicbe. Lerici, 1971, pp. $21-25$.

[ra] Cl. Albore-Livadie. - L'épave étrusque du Cap d'Antibes, Rev. Et. Lig., XXXIII, 1967, 1-3, Hommage à F. Benoit 1,1972 , Pp. 300-326.

[2] Ranuccio BıANCHI-BANDINEL.1. \& Antonio GiUliano. - Etruscbi e Italici prima del dominio di Roma. Rizzoli, Milano, 1973.

[3] Francesca Bortani, Maria Catal.di, Marinella Pasquinuca \& Filippo Conrelli. - Le Citta Etruscbe. Mondadori, 1973.

[4] John BOARDMan. - Atbenian black figure vases. ...

[s] John Bonrdman. - I Greci sui mari. (trad. de l'Anglais). Giunti Martello, Firenze, 1986

[sa] Bernard Bouloumié. - L'épave étrusque d'Antibes, in Voyage en Massalie, Edisud, Marscille, 1990, pp. 42-46.

[6] Julius CAESAR. - De Bello Gallico (L. III, 13, 1).

[7] Lionel CAsson. - Hemiolia and Triemiolia. $J^{a /}$ of Hellenic Studies. LXXVIII, 1958 , pp. 14 sqq.

[8] Mauro Cristofani. - L'arte degli Etruscbi. Torino, 1978.

[9] Mauro Cristofani. - Les Etrusques. Atlas, Paris, 1979.

[10] Ch. Daremberg \& Edm. ShGlio. Dictionnaire des antiquités grecques et romaines. Hachette, Paris, $188 \mathrm{I}$.

[11] Barry FEL.t. - America B.C. Quadrangle, New-York, 1976.

[12] Paolo Giannini. - Centri Etruschi e Romani dell'Etruria Meridionale. Giannini, La Quercia, Viterbo (entre 1981 et 1987).

[13] Paolo Giannini. - Communication personnelle, 1987.

[14] Michel Gras, - Les Etrusques et la mer. Dossiers de l'Arcbéologie, $\mathrm{N}^{\circ} 24$, Sept./Oct. 1977, pp. 45-49.

[14a] Antoinette Hesnard. - Marseille et la mer. Dossiers de I'Archéologie, $\mathrm{N}^{*} 154$, Nov. 1990 , pp. 50-57.

[15] Paul Johnstone. - The Archaeology of Ships. The Bodley Head, London, 1973.

[16] Carlo Maurizio LeRICI. - Le Applicazioni geofisiche alla ricerca archcologica. Studi Etruscbi, 1958, pp. 297-301.

[17] R.E. Linington. - Prove eseguite con il periscopio distanziometrico. Prospezioni archeologicbe. Lerici, 1971, Pp. 33-41.

[17a] Luc LoNG et Florence Richez. - Epave de Bon Porté, in Voyage en Massalie. Edisud, Marseille, 1990, pp. 230-231.

[18] Raniero Mengarel.l. - Caere e le recente scoperte. Studi Etruschi 1, 1927, pp. 145-171.

[19] Mario MoretT1. - La Tomba della Nave. Bollettino d'Arte. Vol. XLV, S. IV, 1960, pp. 346-352.

[20] Mario MORETtr. - La Tomba della Nave. Milano, 1961. (Lerici).

[21] Mario MORETTI. - Nuovi Monumenti della Pittura etrusca. Lerici, Milano, 1966.

[22] Mario MoretTl. - Pittura etrusca in Tarquinia. Milano, 1974.

[23] Mario MORETTI. - Tarquinia. Novara, 1974.

[24] Mario MoretTI. - Cerveteri. Novara, 1977.

[25] Mario Moretti, Guglicmo MaEtzKe \& Manuel Gasser. Art et civilisation des Etrusques. Hachette, Paris, 1970.

[26] Mario MoretTI \& A. ZANer.h. - Givida al Museo di Tarquinia. ...

[27] Sergio PAgL.IERI. - Origine e diffusione delle navi etruscoitaliche. Studi Etruschi. Vol. XXVIII, scric II, 1960, pp. 209-231. 
[28] Massimo Pallotrino, - La peinture étrusque. Skira, Paris, New York, 1952

[29] Massimo Paliottino. - Etruscologia. Hoepli, Milano, 1968 (6 Edition) (Chap. 3 : "Gli Etruschi sul Mare ", pp. 119-137).

[30] Massimo Pallottino, - Proposte, miraggi, perplessità della ricostruzione della storia etrusca. Studi Etruschi. 1987, pp. 3-16.

[31] G. PARENTI. - Il periscopio Nistri per esplorazione archeologica. VII Congresso Internazionale di Arcbeologica classica, Milano, $1958(51-55)$.

[32] G. PARentl. - Nuovo dispositivo distanziometrico per il periscopio Nistri. Prospezioni Arcbéologiche. Lerici, 1971, pp. 29-31.

[32a] Patrice Pomey, - Epaves de commerce et archéologie sousmarine, in Voyage en Massalie. Edisud, Marseille, 1990, pp. 226229 [32b] G. PrUvot, - Epave antique étrusco-punique? an Cap d'Antibes, VT siècle avant Jésus-Cbrist. Antibes, 1971.

[33] René Rebuffat. - Naissance de la marine étrusque. Dossiers de I Archéologie, $\mathrm{N}^{\circ}{ }_{24}$, Sept.-Oct. 1977, pp. 50-57.

[34] Salomon Reinach. - Répertoire des vases grecs et étrusques. Leroux, Paris, 1899 \& 1900 .

[35] Jean Rougé. - La marine dans lantiquité. P.U.F., Paris, 1975.

[36] Steph. Steingraber. - Catalogo ragionato della pittura etrusca. Milano, $19^{84}$.

[37] James Wellard. - The search for the Etruscans. Cardinal, London, 1973.

[38] R.T. WILLinds. - Early Greek Ships of two levels. $J^{a l}$ of Hellenic Studies, LXXVIII, 1958, pp. 121-130 \& pl. XIII-XV.

\section{La tomba della nave di Tarquinia, \\ e la nave etrusca di carico}

La scoperta, dovuta all'uso di metodi di prospezione molto elaborati. di una tomba etrusca affrescata che mostrava, quasi 2s secoli fa, una grande nave di commercio, ci offre informazioni preziose sulla marina tirrena all'epoca del suo apice e subito dopo.

Quella unica rappresentazione, confrontata alle figure di navi dei paesi commercianti vicini, ai relitti ed ai loro carichi, ai testi antichi, ci permette di migliorare la nostra conoscenza del commercio maritimo etrusco e delle navi di carico, in un contesto geografico in cui, per molto tempo, "il mare nostro » fu il legame più efficace. 